\title{
BOUNDEDNESS OF THE MAXIMAL OPERATOR AND ITS COMMUTATORS ON VANISHING GENERALIZED ORLICZ-MORREY SPACES
}

\author{
Fatih Deringoz, Vagif S. Guliyev and Stefan Samko
}

Ahi Evran University, Department of Mathematics

Bagbasi Campus, 40100, Kirsehir, Turkey; fderingoz@ahievran.edu.tr

Ahi Evran University, Department of Mathematics

Bagbasi Campus, 40100, Kirsehir, Turkey, and Institute of Mathematics and Mechanics

B. Vahabzade 9, AZ1141, Baku, Azerbaijan; vagif@guliyev.com

Universidade do Algarve, Departamento de Matemática,

Campus de Gambelas, 8005-139 Faro, Portugal; ssamko@ualg.pt

\begin{abstract}
We prove the boundedness of the Hardy-Littlewood maximal operator and their commutators with BMO-coefficients in vanishing generalized Orlicz-Morrey spaces $V \mathcal{M}^{\Phi, \varphi}\left(\mathbf{R}^{n}\right)$ including weak versions of these spaces. The main advance in comparison with the existing results is that we manage to obtain conditions for the boundedness not in integral terms but in less restrictive terms of supremal operators involving the Young function $\Phi(u)$ and the function $\varphi(x, r)$ defining the space. No kind of monotonicity condition on $\varphi(x, r)$ in $r$ is imposed.
\end{abstract}

\section{Introduction}

1.1. Some background. As is well known, Morrey spaces are widely used to investigate the local behavior of solutions to second order elliptic partial differential equations (PDE). Recall that the classical Morrey spaces $\mathcal{M}^{p, \lambda}\left(\mathbf{R}^{n}\right)$ have an origin in [23] and are defined by

$$
\mathcal{M}^{p, \lambda}\left(\mathbf{R}^{n}\right)=\left\{f \in L_{\mathrm{loc}}^{p}\left(\mathbf{R}^{n}\right):\|f\|_{\mathcal{M}^{p, \lambda}}:=\sup _{x \in \mathbf{R}^{n}, r>0} r^{-\frac{\lambda}{p}}\|f\|_{L^{p}(B(x, r))}<\infty\right\},
$$

where $0 \leq \lambda \leq n, 1 \leq p<\infty$. Here and everywhere in the sequel $B(x, r)$ is the ball in $\mathbf{R}^{n}$ of radius $r$ centered at $x$ and $|B(x, r)|$ stands for its Lebesgue measure.

By $W \mathcal{M}^{p, \lambda} \equiv W \mathcal{M}^{p, \lambda}\left(\mathbf{R}^{n}\right)$ we denote the weak Morrey space defined as the set of functions $f \in W L_{\text {loc }}^{p}\left(\mathbf{R}^{n}\right)$ for which

$$
\|f\|_{W \mathcal{M}^{p, \lambda}}=\sup _{x \in \mathbf{R}^{n}, r>0} r^{-\frac{\lambda}{p}}\|f\|_{W L^{p}(B(x, r))}<\infty .
$$

doi:10.5186/aasfm.2015.4029

2010 Mathematics Subject Classification: Primary 42B20, 42B25, 42B35, 46E30.

Key words: Vanishing generalized Orlicz-Morrey space, Hardy-Littlewood maximal operator, singular integral operators, commutator, BMO.

The research of V. Guliyev and F. Deringoz was partially supported by the grant of Ahi Evran University Scientific Research Projects (PYO.FEN.4003.13.003) and (PYO.FEN.4001.14.017). The research of V. Guliyev is partially supported by the grant of Science Development Foundation under the President of the Republic of Azerbaijan, Grant EIF-2013-9(15)-46/10/1. In the case of S. Samko the research was supported by the grant no. 15-01-02732 of Russian Fund of Basic Research. 
Without danger of confusion of notation, by $\mathcal{M}^{p, \varphi}$ we denote the generalized Morrey space defined by the norm

$$
\|f\|_{\mathcal{M}^{p, \varphi}}:=\sup _{x \in \mathbf{R}^{n}, r>0} \frac{1}{\varphi(x, r)}\|f\|_{L^{p}(B(x, r))}
$$

where $\varphi$ is a measurable function positive on $\mathbf{R}^{n} \times(0, \infty)$.

Orlicz spaces are useful tools in harmonic analysis and its applications. For example, the Hardy-Littlewood maximal operator

$$
M f(x)=\sup _{r>0} \frac{1}{|B(x, r)|} \int_{B(x, r)}|f(y)| d y
$$

is bounded on $L^{p}$ for $1<p \leq \infty$, but not on $L^{1}$, but via Orlicz spaces, we can study its boundedness near $p=1$, see $[18,19]$ and [4] for precise statements.

1.2. On Orlicz-Morrey spaces and the goal of the paper. A natural step in the theory of functions spaces was to study Orlicz-Morrey spaces

$$
\mathcal{M}^{\Phi, \varphi}\left(\mathbf{R}^{n}\right)
$$

where the "Morrey-type measuring" of regularity of functions is realized with respect to the Orlicz norm over balls instead of the Lebesgue one. Such spaces were studied in [24] first, see also [7, 13, 25, 26, 34]. The most general spaces of such a type, MusielakOrlicz-Morrey spaces, unifying the classical and variable exponent approaches, were an object of study in [22], where potential operators were studied.

The Orlicz-Morrey spaces we work with are precisely defined in Section 2.3. The weakest assumptions on the functions $\Phi$ and $\varphi$, defining the space $\mathcal{M}^{\Phi, \varphi}\left(\mathbf{R}^{n}\right)$, for the boundedness of the maximal operator, were obtained in [7], up to authors' knowledge, together with weak estimates.

The definition of Orlicz-Morrey spaces introduced in [7] and used here is different from that of the papers [24], [34] and other papers.

Morrey and Orlicz-Morrey spaces are not separable due to the $L^{\infty}$-norm with respect to $r$ and $x$. The closure of nice functions in the Morrey or Orlicz-Morrey norm gives a subspace of the corresponding space. Such a subspace corresponding to the classical Morrey space $\mathcal{M}^{p, \lambda}$, known under the name of vanishing Morrey space, appeared in connection with PDE in [36], [37], they were also used in [28]. The vanishing generalized Morrey spaces were introduced and studied [30], see also a study of commutators of Hardy operators in such spaces in [27].

Note that commutators in Morrey spaces were studied in a less generality in comparison with other spaces. In the case of the classical Morrey spaces we refer for instance to [28] and [32], in the case of generalized Morrey spaces to [35] (where some monotonicity assumptions were imposed on the function $\varphi$, but on the other hand an anisotropic case was admitted) and to $[14,15]$ (where no monotonicity assumptions on $\varphi$ were imposed, including an anisotropic case [15]), where other references may be also found. The results for commutators in the setting of Orlicz-Morrey spaces in this paper are new even in the case of non-vanishing spaces.

Vanishing Orlicz-Morrey spaces, including their weak versions appeared in [13], where there was studied the boundedness of the so called $\Phi$-admissible sublinear operators and their commutators. The notion of $\Phi$-admissible sublinear operators generalizes the notion of $p$-admissible operators corresponding to the case $\Phi(u)=u^{p}$, 
introduced in [30], where the boundedness of $p$-admissible operators of singular type in vanishing generalized Morrey spaces was studied.

The result obtained in [13] for the maximal operator and its commutators provides boundedness conditions in terms of some integral inequality.

The main goal of this paper is to show that the boundedness of the maximal operator and its commutators in vanishing Orlicz-Morrey spaces may be obtained under weaker conditions, namely in terms of the so called supremal operators. More precisely, we find sufficient conditions, in supremal terms, on the Young function $\Phi$ and functions $\varphi_{1}, \varphi_{2}$ which ensure the boundedness of the maximal operator and its commutators from one vanishing generalized Orlicz-Morrey space $V \mathcal{M}^{\Phi, \varphi_{1}}\left(\mathbf{R}^{n}\right)$ to another $V \mathcal{M}^{\Phi, \varphi_{2}}\left(\mathbf{R}^{n}\right)$, including weak estimates.

Finally note that there is a close relations between of vanishing Morrey spaces and so called Stummel classes. They are different, but are closely embedded into each other: every vanishing Morrey space is embedded between two very close to each other Stummel classes and vice versa. Such relations were studied in [31] in the case $\Phi(u)=u^{p}$. We do not touch such a study for the general case of Orlicz spaces in this paper.

1.3. On some operators. It is well-known that commutators of classical operators of harmonic analysis play an important role in various topics of analysis and PDE, see for instance [3], [6] and [5], where in particular in [6] it was shown that the commutator $[b, T] f=T(b f)-b T f$ of the Calderón-Zygmund operator $T$ with $b \in B M O\left(\mathbf{R}^{n}\right)$ is bounded on $L^{p}\left(\mathbf{R}^{n}\right)$ for $1<p<\infty$.

In this paper, in the setting of Orlicz-Morrey spaces, we consider the commutator $\mathfrak{M}^{b}(f)$ of the maximal operator defined by

$$
\mathfrak{M}^{b}(f)(x)=\sup _{t>0} \frac{1}{|B(x, t)|} \int_{B(x, t)}|b(x)-b(y)||f(y)| d y
$$

studied in [9] in the space $L^{p}\left(\mathbf{R}^{n}\right)$.

The definitions related to the so called $\Phi$-admissible operators, containing the maximal operator as a particular case, may be found in [13]. However we provide the corresponding details here for completeness of presentation, keeping in mind that we wish to compare the obtained results with those in [13].

Let $T$ be a sublinear operator, i.e.,

$$
|T(f+g)| \leq|T f|+|T g| .
$$

In the definition of $\Phi$-admissible operator of singular type given below we follow [30], where this notion was first introduced in the case $\Phi(u)=u^{p}$

Definition 1.1. ( $\Phi$-admissible operator of singular type) Let $\Phi$ be a Young function. A sublinear operator $T$ will be called $\Phi$-admissible operator of singular type or respectively weakly $\Phi$-admissible operator of singular type, if:

1) $T$ satisfies the size condition of the form

$$
\chi_{B(x, r)}(z)\left|T\left(f \chi_{\mathbf{R}^{n} \backslash B(x, 2 r)}\right)(z)\right| \leq C \chi_{B(x, r)}(z) \int_{\mathbf{R}^{n} \backslash B(x, 2 r)} \frac{|f(y)|}{|y-z|^{n}} d y
$$

for $x \in \mathbf{R}^{n}$, a.e. $z \in \mathbf{R}^{n}$ and $r>0$;

2) $T$ is bounded from $L^{\Phi}\left(\mathbf{R}^{n}\right)$ to $L^{\Phi}\left(\mathbf{R}^{n}\right)$, or to the weak space $W L^{\Phi}\left(\mathbf{R}^{n}\right)$, respectively. 
Definition 1.2. ( $\Phi$-admissible commutators of operators of singular type) Let $\Phi$ be a Young function and $T$ a sublinear operator. The operator $T_{b}$ depending on a function $b$, will be called $\Phi$-admissible commutator of a singular type operator $T$, if:

1) $T_{b}$ satisfies the size condition of the form

$$
\begin{aligned}
& \chi_{B(x, r)}(z)\left|T_{b}\left(f \chi_{\mathbf{R}^{n} \backslash B(x, 2 r)}\right)(z)\right| \leq C \chi_{B(x, r)}(z) \int_{\mathbf{R}^{n} \backslash B(x, 2 r)} \frac{|b(y)-b(z)||f(y)|}{|y-z|^{n}} d y \\
& \quad \text { for } x \in \mathbf{R}^{n} \text {, a.a. } z \in \mathbf{R}^{n} \text { and } r>0 \text {; } \\
& \text { 2) } T_{b} \text { is bounded in } L^{\Phi}\left(\mathbf{R}^{n}\right) \text {. }
\end{aligned}
$$

\section{Preliminaries}

We refer to the books $[20,21,29]$ for the theory of Orlicz spaces, but provide some basic definitions and facts to be precise in formulations. A function $\Phi:[0,+\infty) \rightarrow$ $[0, \infty]$ is called a Young function if it is convex, left-continuous, $\lim _{r \rightarrow+0} \Phi(r)=\Phi(0)=0$ and $\lim _{r \rightarrow+\infty} \Phi(r)=\infty$. Any Young function is increasing. Let

$$
\Phi^{-1}(s)=\inf \{r \geq 0: \Phi(r)>s\}, \quad 0 \leq s \leq+\infty .
$$

It is known that

$$
\Phi\left(\Phi^{-1}(r)\right) \leq r \leq \Phi^{-1}(\Phi(r)) \text { for } 0 \leq r<+\infty .
$$

Recall that the $\Delta_{2}$-condition, denoted also as $\Phi \in \Delta_{2}$, is $\Phi(2 r) \leq k \Phi(r)$, and the $\nabla_{2}$-condition, denoted also by $\Phi \in \nabla_{2}$, is $\Phi(r) \leq \frac{1}{2 k} \Phi(k r), r \geq 0$, where $k>1$.

The function

$$
\widetilde{\Phi}(r)= \begin{cases}\sup \{r s-\Phi(s): s \in[0, \infty)\}, & r \in[0, \infty) \\ +\infty, & r=+\infty\end{cases}
$$

complementary to a Young function $\Phi$, is also a Young function and $\widetilde{\widetilde{\Phi}}=\Phi$.

A Young function $\Phi$ is said to be of upper type $p$ (resp. lower type $p$ ), $p \in[0, \infty)$, if for all $t \in[1, \infty)$ (resp. $t \in[0,1])$ and $s \in[0, \infty), \Phi(t s) \leq C t^{p} \Phi(s)$.

Remark 2.1. It is known [20] that for a Young function $\Phi$ to be of lower type $p_{0}$ and upper type $p_{1}$ with $1<p_{0} \leq p_{1}<\infty$, is equivalent to $\Phi \in \Delta_{2} \cap \nabla_{2}$.

We will also use the numerical characteristics

$$
a_{\Phi}:=\inf _{t \in(0, \infty)} \frac{t \Phi^{\prime}(t)}{\Phi(t)}, \quad b_{\Phi}:=\sup _{t \in(0, \infty)} \frac{t \Phi^{\prime}(t)}{\Phi(t)}
$$

of Young functions.

Remark 2.2. It is known that $\Phi \in \Delta_{2} \cap \nabla_{2}$ if and only if $1<a_{\Phi} \leq b_{\Phi}<\infty$, see [21]. Then as a consequence of Remark 2.1, a Young function $\Phi$ is of lower type $p_{0}$ and upper type $p_{1}$ with $1<p_{0} \leq p_{1}<\infty$ if and only if $1<a_{\Phi} \leq b_{\Phi}<\infty$.

The Orlicz space $L^{\Phi}\left(\mathbf{R}^{n}\right)$ everywhere in the sequel is defined by a Young function $\Phi$ via the norm

$$
\|f\|_{L^{\Phi}}=\inf \left\{\lambda>0: \int_{\mathbf{R}^{n}} \Phi\left(\frac{|f(x)|}{\lambda}\right) d x \leq 1\right\}
$$


and the weak Orlicz space by

$$
\|f\|_{W L^{\Phi}}=\inf \left\{\lambda>0: \sup _{t>0} \Phi(t)\left|\left\{x \in \mathbf{R}^{n}:|f(x)|>\lambda t\right\}\right| \leq 1\right\} .
$$

The notation $L_{\text {loc }}^{\Phi}\left(\mathbf{R}^{n}\right)$ will stand for the set of functions $f$ such that $f \chi_{B} \in L^{\Phi}\left(\mathbf{R}^{n}\right)$ for all balls $B \subset \mathbf{R}^{n}$.

2.1. Some supremal inequalities. Let $v$ be a weight and $L_{v}^{\infty}(0, \infty)$ the space defined by the norm $\|g\|_{L_{v}^{\infty}(0, \infty)}:=\sup _{t>0} v(t)|g(t)|$. Let $\mathfrak{M}(0, \infty)$ be the set of all Lebesgue-measurable functions on $(0, \infty)$, and $\mathfrak{M}^{+}(0, \infty)$ its subset of nonnegative functions on $(0, \infty)$ and $\mathfrak{M}^{+}(0, \infty ; \uparrow)$ be the cone of non-decreasing functions in $\mathfrak{M}^{+}(0, \infty)$. Denote also

$$
\mathcal{A}=\left\{\varphi \in \mathfrak{M}^{+}(0, \infty ; \uparrow): \lim _{t \rightarrow 0+} \varphi(t)=0\right\} .
$$

Let $u$ be a continuous and non-negative function on $(0, \infty)$. We define the supremal operator $\bar{S}_{u}$ on $g \in \mathfrak{M}(0, \infty)$ by

$$
\left(\bar{S}_{u} g\right)(r):=\left\|\left(1+\ln \frac{t}{r}\right) u(t) g(t)\right\|_{L^{\infty}(r, \infty)}, \quad r \in(0, \infty) .
$$

The following theorem is proved analogously to Lemma 5.2 in [2].

Theorem 2.3. Let $v_{1}$ and $v_{2}$ be weights and $0<\left\|v_{1}\right\|_{L^{\infty}(t, \infty)}<\infty$ for any $t>0$ and let $u$ be a continuous non-negative function on $(0, \infty)$. Then the operator $\bar{S}_{u}$ is bounded from $L_{v_{1}}^{\infty}(0, \infty)$ to $L_{v_{2}}^{\infty}(0, \infty)$ on the cone $\mathcal{A}$ if and only if

$$
\left\|v_{2} \bar{S}_{u}\left(\left\|v_{1}\right\|_{L^{\infty}(\cdot, \infty)}^{-1}\right)\right\|_{L^{\infty}(0, \infty)}<\infty
$$

2.2. On boundedness of the maximal operator and their commutators in Orlicz spaces. The following lemma was proved in [7].

Lemma 2.4. Let $f \in L_{\text {loc }}^{\Phi}\left(\mathbf{R}^{n}\right)$. Then for Young functions $\Phi \in \nabla_{2}$

$$
\|M f\|_{L^{\Phi}(B(x, r))} \lesssim \frac{1}{\Phi^{-1}\left(r^{-n}\right)} \sup _{t>2 r} \Phi^{-1}\left(t^{-n}\right)\|f\|_{L^{\Phi}(B(x, t))}
$$

and for any Young function $\Phi$

$$
\|M f\|_{W L^{\Phi}(B(x, r))} \lesssim \frac{1}{\Phi^{-1}\left(r^{-n}\right)} \sup _{t>2 r} \Phi^{-1}\left(t^{-n}\right)\|f\|_{L^{\Phi}(B(x, t))} .
$$

Conditions for the boundedness of the maximal operator in Orlicz spaces are known, see [10], [20]; we use such a result in the theorem below in the form proved in [7].

Theorem 2.5. Let $\Phi$ be a Young function. Then the maximal operator $M$ is bounded from $L^{\Phi}\left(\mathbf{R}^{n}\right)$ to $W L^{\Phi}\left(\mathbf{R}^{n}\right)$. If $\Phi \in \nabla_{2}$, it is bounded in $L^{\Phi}\left(\mathbf{R}^{n}\right)$.

The known boundedness statements for the commutator operator $\mathfrak{M}^{b}$ on Orlicz spaces run as follows, see [8, Theorem 1.9 and Corollary 2.3]. Note that in [8] a more general case of multi-linear commutators was studied. 
We recall that the space $B M O\left(\mathbf{R}^{n}\right)=\left\{b \in L_{\text {loc }}^{1}\left(\mathbf{R}^{n}\right):\|b\|_{*}<\infty\right\}$ is defined by the seminorm

$$
\|b\|_{*}:=\sup _{x \in \mathbf{R}^{n}, r>0} \frac{1}{|B(x, r)|} \int_{B(x, r)}\left|b(y)-b_{B(x, r)}\right| d y<\infty
$$

where $b_{B(x, r)}=\frac{1}{|B(x, r)|} \int_{B(x, r)} b(y) d y$. We will need the following properties of BMOfunctions:

$$
\|b\|_{*} \approx \sup _{x \in \mathbf{R}^{n}, r>0}\left(\frac{1}{|B(x, r)|} \int_{B(x, r)}\left|b(y)-b_{B(x, r)}\right|^{p} d y\right)^{\frac{1}{p}}
$$

where $1 \leq p<\infty$, and

$$
\left|b_{B(x, r)}-b_{B(x, t)}\right| \leq C\|b\|_{*} \ln \frac{t}{r} \quad \text { for } 0<2 r<t,
$$

where $C$ does not depend on $b, x, r$ and $t$.

Theorem 2.6. Let $\Phi$ be a Young function with $1<a_{\Phi} \leq b_{\Phi}<\infty$ and $b \in$ $B M O\left(\mathbf{R}^{n}\right)$. Then $\mathfrak{M}^{b}$ is bounded on $L^{\Phi}\left(\mathbf{R}^{n}\right)$.

\subsection{Generalized Orlicz-Morrey space.}

Definition 2.7. [7] Let $\varphi(x, r)$ be a positive measurable function on $\mathbf{R}^{n} \times(0, \infty)$ and $\Phi$ any Young function. The generalized Orlicz-Morrey space $\mathcal{M}^{\Phi, \varphi}\left(\mathbf{R}^{n}\right)$ is the space of functions $f \in L_{\text {loc }}^{\Phi}\left(\mathbf{R}^{n}\right)$ with the finite norm

$$
\|f\|_{\mathcal{M}^{\Phi, \varphi}}=\sup _{x \in \mathbf{R}^{n}, r>0} \frac{\|f\|_{L^{\Phi}(B(x, r))}}{\varphi(x, r)},
$$

and the weak generalized Orlicz-Morrey space $W \mathcal{M}^{\Phi, \varphi}\left(\mathbf{R}^{n}\right)$ as the set of functions $f \in W L_{\mathrm{loc}}^{\Phi}\left(\mathbf{R}^{n}\right)$ for which

$$
\|f\|_{W \mathcal{M}^{\Phi, \varphi}}=\sup _{x \in \mathbf{R}^{n}, r>0} \frac{\|f\|_{W L^{\Phi}(B(x, r))}}{\varphi(x, r)}<\infty .
$$

Clearly, we recover the spaces $\mathcal{M}^{p, \varphi}$ and $W \mathcal{M}^{p, \varphi}$ under the choice $\Phi(r)=r^{p}, 1 \leq$ $p<\infty$.

The following theorem was proved in [7]. Our results for vanishing spaces are based on this theorem.

Theorem 2.8. Let $\Phi$ be a Young function, the functions $\varphi_{1}, \varphi_{2}$ and $\Phi$ satisfy the condition

$$
\sup _{r<t<\infty} \operatorname{ess~inf}_{t<s<\infty} \varphi_{1}(x, s) \Phi^{-1}\left(t^{-n}\right) \leq C \varphi_{2}(x, r) \Phi^{-1}\left(r^{-n}\right),
$$

where $C$ does not depend on $x$ and $r$. Then the maximal operator $M$ is bounded from $\mathcal{M}^{\Phi, \varphi_{1}}\left(\mathbf{R}^{n}\right)$ to $W \mathcal{M}^{\Phi, \varphi_{2}}\left(\mathbf{R}^{n}\right)$ for any Young function $\Phi$ and from $\mathcal{M}^{\Phi, \varphi_{1}}\left(\mathbf{R}^{n}\right)$ to $\mathcal{M}^{\Phi, \varphi_{2}}\left(\mathbf{R}^{n}\right)$ for $\Phi \in \nabla_{2}$.

In the case $\Phi(t)=t^{p}$, from Theorem 2.8 we get the following corollary, which is proven in [1] on $\mathbf{R}^{n}$.

Corollary 2.9. Let $1 \leq p<\infty$ and $\left(\varphi_{1}, \varphi_{2}\right)$ satisfies the condition

$$
\sup _{r<t<\infty} \frac{\underset{t<s<\infty}{\operatorname{ess} \inf } \varphi_{1}(x, s)}{t^{\frac{n}{p}}} \leq C \frac{\varphi_{2}(x, r)}{r^{\frac{n}{p}}}
$$


where $C$ does not depend on $x$ and $r$. Then for $p>1, M$ is bounded from $\mathcal{M}^{p, \varphi_{1}}\left(\mathbf{R}^{n}\right)$ to $\mathcal{M}^{p, \varphi_{2}}\left(\mathbf{R}^{n}\right)$ and for $p=1, M$ is bounded from $\mathcal{M}^{1, \varphi_{1}}\left(\mathbf{R}^{n}\right)$ to $W \mathcal{M}^{1, \varphi_{2}}\left(\mathbf{R}^{n}\right)$.

Note that the result of Corollary 2.9 is stronger than the Euclidean version of a result for the maximal operator in Lebesgue-Morrey spaces (the case $\Phi(u)=u^{p}$ ) obtained in [33], where the general underlying quasi-metric measure space $(X, d \mu)$ was admitted. In the case where $X=\mathbf{R}^{n}$ and $\mu$ is the Lebesgue measure, the result from [33] runs as follows.

Theorem 2.10. [33, Theorem 2.3] Let $p \in[1, \infty)$ and let $\varphi:(0, \infty) \rightarrow(0, \infty)$ be an increasing function. Assume that the mapping $r \mapsto \frac{\varphi(r)}{r^{\frac{n}{p}}}$ is almost decreasing (there exists a constant $c$ such that for $s<r$ we have $\left.\frac{\varphi(r)}{r^{\frac{n}{p}}} \leq c \frac{\varphi(s)}{s^{\frac{n}{p}}}\right)$. Then there exists a constant $C>0$ such that

$$
\|M f\|_{\mathcal{M}^{p, \varphi}} \leq C\|f\|_{\mathcal{M}^{p, \varphi}} \text { if } p>1
$$

and

$$
\|M f\|_{W \mathcal{M}^{1, \varphi}} \leq C\|f\|_{\mathcal{M}^{1, \varphi}}
$$

\section{Vanishing generalized Orlicz-Morrey spaces}

Definition 3.1. The vanishing generalized Orlicz-Morrey space $V \mathcal{M}^{\Phi, \varphi}\left(\mathbf{R}^{n}\right)$ and its weak version $V W \mathcal{M}^{\Phi, \varphi}\left(\mathbf{R}^{n}\right)$ are defined as the spaces of functions $f \in$ $\mathcal{M}^{\Phi, \varphi}\left(\mathbf{R}^{n}\right)$ and $f \in W \mathcal{M}^{\Phi, \varphi}\left(\mathbf{R}^{n}\right)$ such that

$$
\lim _{r \rightarrow 0} \sup _{x \in \mathbf{R}^{n}} \frac{\|f\|_{L^{\Phi}(B(x, r))}}{\varphi(x, r)}=0 \quad \text { and } \quad \lim _{r \rightarrow 0} \sup _{x \in \mathbf{R}^{n}} \frac{\|f\|_{W L^{\Phi}(B(x, r))}}{\varphi(x, r)}=0,
$$

respectively.

Conditions sufficient for the non-triviality of the spaces $V \mathcal{M}^{\Phi, \varphi}\left(\mathbf{R}^{n}\right)$ and $V W \mathcal{M}^{\Phi, \varphi}\left(\mathbf{R}^{n}\right)$ have the form

$$
\lim _{r \rightarrow 0} \frac{1}{\Phi^{-1}\left(r^{-n}\right) \varphi(x, r)}=0
$$

and

$$
\sup _{0<r<\infty} \frac{1}{\Phi^{-1}\left(r^{-n}\right) \varphi(x, r)}<\infty
$$

respectively, because bounded functions with compact support belong then to these spaces; in the case $\Phi(u)=u^{p}$ these conditions appeared in [30].

The spaces $V \mathcal{M}^{\Phi, \varphi}\left(\mathbf{R}^{n}\right)$ and $V W \mathcal{M}^{\Phi, \varphi}\left(\mathbf{R}^{n}\right)$ are closed subspaces of the Banach spaces $\mathcal{M}^{\Phi, \varphi}\left(\mathbf{R}^{n}\right)$ and $W \mathcal{M}^{\Phi, \varphi}\left(\mathbf{R}^{n}\right)$, respectively, which may be shown by standard means.

We will also use the notation

$$
\mathfrak{A}_{\Phi, \varphi}(f ; x, r):=\frac{\|f\|_{L^{\Phi}(B(x, r))}}{\varphi(x, r)} \text { and } \quad \mathfrak{A}_{\Phi, \varphi}^{W}(f ; x, r):=\frac{\|f\|_{W L^{\Phi}(B(x, r))}}{\varphi(x, r)}
$$

for brevity, so that

$$
V \mathcal{M}^{\Phi, \varphi}\left(\mathbf{R}^{n}\right)=\left\{f \in \mathcal{M}^{\Phi, \varphi}\left(\mathbf{R}^{n}\right): \lim _{r \rightarrow 0} \sup _{x \in \mathbf{R}^{n}} \mathfrak{A}_{\Phi, \varphi}(f ; x, r)=0\right\}
$$

and similarly for $V W \mathcal{M}^{\Phi, \varphi}\left(\mathbf{R}^{n}\right)$. 


\section{Boundedness of the maximal operator in the spaces $V \mathcal{M}^{\Phi, \varphi}\left(\mathbf{R}^{n}\right)$}

In this section we give sufficient conditions on $\Phi$ and $\varphi$ for the boundedness of the maximal operator $M$ in vanishing generalized Orlicz-Morrey spaces $V \mathcal{M}^{\Phi, \varphi}\left(\mathbf{R}^{n}\right)$.

Theorem 4.1. Let $\Phi$ be a Young function, $\varphi_{2}$ satisfy (3.1), the functions $\varphi_{1}, \varphi_{2}$ and $\Phi$ satisfy the conditions

$$
m_{\delta}:=\sup _{\delta<t<\infty} \sup _{x \in \mathbf{R}^{n}} \varphi_{1}(x, t) \Phi^{-1}\left(t^{-n}\right)<\infty
$$

for every $\delta>0$ and the supremal condition

$$
\frac{\sup _{r<t<\infty} \Phi^{-1}\left(t^{-n}\right) \varphi_{1}(x, t)}{\Phi^{-1}\left(r^{-n}\right) \varphi_{2}(x, r)} \leq C_{0},
$$

where $C_{0}$ does not depend on $x$ and $r$. Then the maximal operator $M$ is bounded from $V \mathcal{M}^{\Phi, \varphi_{1}}\left(\mathbf{R}^{n}\right)$ to $V W \mathcal{M}^{\Phi, \varphi_{2}}\left(\mathbf{R}^{n}\right)$ and, if $\Phi \in \nabla_{2}$, from $V \mathcal{M}^{\Phi, \varphi_{1}}\left(\mathbf{R}^{n}\right)$ to $V \mathcal{M}^{\Phi, \varphi_{2}}\left(\mathbf{R}^{n}\right)$.

Proof. The norm inequalities follow from Theorem 2.8, so we only have to prove that

$$
\lim _{r \rightarrow 0} \sup _{x \in \mathbf{R}^{n}} \mathfrak{A}_{\Phi, \varphi_{1}}(f ; x, r)=0 \Longrightarrow \lim _{r \rightarrow 0} \sup _{x \in \mathbf{R}^{n}} \mathfrak{A}_{\Phi, \varphi_{2}}(M f ; x, r)=0,
$$

when $\Phi \in \nabla_{2}$, and

$$
\lim _{r \rightarrow 0} \sup _{x \in \mathbf{R}^{n}} \mathfrak{A}_{\Phi, \varphi_{1}}(f ; x, r)=0 \Longrightarrow \lim _{r \rightarrow 0} \sup _{x \in \mathbf{R}^{n}} \mathfrak{A}_{\Phi, \varphi_{2}}^{W}(M f ; x, r)=0
$$

otherwise. In the justification of the above passage to the limit we follow ideas of [30], where the case $\Phi(r)=r^{p}$ was considered, but base ourselves on Lemma 2.4.

We start with (4.3). We rewrite the inequality (2.2) in the form

$$
\mathfrak{A}_{\Phi, \varphi_{2}}(M f ; x, r) \leq C \frac{\sup _{t>r} \Phi^{-1}\left(t^{-n}\right)\|f\|_{L^{\Phi}(B(x, t))}}{\varphi_{2}(x, r) \Phi^{-1}\left(r^{-n}\right)} .
$$

To show that $\sup _{x \in \mathbf{R}^{n}} \mathfrak{A}_{\Phi, \varphi_{2}}(M f ; x, r)<\varepsilon$ for small $\mathrm{r}$, we split the right-hand side of (4.5):

$$
\mathfrak{A}_{\Phi, \varphi_{2}}(M f ; x, r) \leq C\left[I_{\delta_{0}}(x, r)+J_{\delta_{0}}(x, r)\right],
$$

where $\delta_{0}>0$ will be chosen as shown below (we may take $\delta_{0}<1$ ),

$$
\begin{aligned}
& I_{\delta_{0}}(x, r):=\frac{\sup _{r<t<\delta_{0}} \Phi^{-1}\left(t^{-n}\right)\|f\|_{L^{\Phi}(B(x, t))}}{\varphi_{2}(x, r) \Phi^{-1}\left(r^{-n}\right)}, \\
& J_{\delta_{0}}(x, r):=\frac{\sup _{t>\delta_{0}} \Phi^{-1}\left(t^{-n}\right)\|f\|_{L^{\Phi}(B(x, t))}}{\varphi_{2}(x, r) \Phi^{-1}\left(r^{-n}\right)}
\end{aligned}
$$

and it is supposed that $r<\delta_{0}$. Now we choose any fixed $\delta_{0}>0$ such that

$$
\sup _{x \in \mathbf{R}^{n}} \mathfrak{A}_{\Phi, \varphi_{1}}(f ; x, t)<\frac{\varepsilon}{2 C C_{0}}, \quad \text { for all } 0<t<\delta_{0},
$$

where $C$ and $C_{0}$ are constants from (4.6) and (4.2), which is possible since $f \in$ $V \mathcal{M}^{\Phi, \varphi_{1}}\left(\mathbf{R}^{n}\right)$. Then $\|f\|_{L^{\Phi}(B(x, t))}<\frac{\varepsilon}{2 C C_{0}} \varphi_{1}(x, t)$ and we obtain the estimate of the first term uniform in $r \in\left(0, \delta_{0}\right)$ :

$$
\sup _{x \in \mathbf{R}^{n}} C I_{\delta_{0}}(x, r)<\frac{\varepsilon}{2}, \quad 0<r<\delta_{0}
$$


by (4.2).

The estimation of the second term now may be made already by the choice of $r$ sufficiently small thanks to the condition (3.1). We have

$$
J_{\delta}(x, r) \leq \frac{m_{\delta_{0}}\|f\|_{\mathcal{M}^{\Phi, \varphi_{1}}}}{\Phi^{-1}\left(r^{-n}\right) \varphi_{2}(x, r)},
$$

where $m_{\delta_{0}}$ is the constant from (4.1) with $\delta=\delta_{0}$. Then, by (3.1) it suffices to choose $r$ small enough such that

$$
\sup _{x \in \mathbf{R}^{n}} \frac{1}{\Phi^{-1}\left(r^{-n}\right) \varphi(x, r)} \leq \frac{\varepsilon}{2 m_{\delta_{0}}\|f\|_{\mathcal{M}^{\Phi, \varphi}}},
$$

which completes the proof of (4.3).

The proof of (4.4) is, line by line, similar to the proof of (4.3).

To compare, we formulate the following theorem proved in [13] and remarks below.

Theorem 4.2. Let $\Phi$ be a Young function, $\varphi_{2}$ satisfy (3.1), the functions $\varphi_{1}, \varphi_{2}$ and $\Phi$ satisfy the conditions

$$
c_{\delta}:=\int_{\delta}^{\infty} \sup _{x \in \mathbf{R}^{n}} \varphi_{1}(x, t) \frac{\Phi^{-1}\left(t^{-n}\right)}{t} d t<\infty
$$

for every $\delta>0$, and

$$
\frac{1}{\varphi_{2}(x, r) \Phi^{-1}\left(r^{-n}\right)} \int_{r}^{\infty} \varphi_{1}(x, t) \Phi^{-1}\left(t^{-n}\right) \frac{d t}{t} \leq C_{0},
$$

where $C_{0}$ does not depend on $x \in \mathbf{R}^{n}$ and $r>0$. Then a $\Phi$-admissible singular operator $T$ is bounded from $V \mathcal{M}^{\Phi, \varphi_{1}}\left(\mathbf{R}^{n}\right)$ to $V \mathcal{M}^{\Phi, \varphi_{2}}\left(\mathbf{R}^{n}\right)$ and a weak $\Phi$-admissible singular operator $T$ is bounded from $V \mathcal{M}^{\Phi, \varphi_{1}}\left(\mathbf{R}^{n}\right)$ to $V W \mathcal{M}^{\Phi, \varphi_{2}}\left(\mathbf{R}^{n}\right)$.

Remark 4.3. The condition (4.7) may be omitted when $\varphi_{1}(x, r)$ does not depend on $x$, since (4.7) follows from (4.8) in this case.

Remark 4.4. As shown in [7], the condition (4.2) is weaker than (4.8): the latter implies the former, while the functions $\varphi_{1}(x, t)=\varphi_{2}(x, t)=\frac{1}{\Phi^{-1}\left(t^{-n}\right)}$ satisfy (4.2), but not (4.8).

\section{On commutators in the spaces $\mathcal{M}^{\Phi, \varphi}\left(\mathrm{R}^{n}\right)$ and $V \mathcal{M}^{\Phi, \varphi}\left(\mathrm{R}^{n}\right)$}

5.1. $\Phi$-admissible commutators of singular type operators. In this subsection, for a possibility to compare, we formulate the following two theorems, where $T_{b}$ is a $\Phi$-admissible commutator of a singular type operator $T$, which were proved in [13].

Theorem 5.1. Let $\Phi$ be a Young function with $1<a_{\Phi} \leq b_{\Phi}<\infty$ and $b \in$ $B M O\left(\mathbf{R}^{n}\right)$. If

$$
\int_{r}^{\infty}\left(1+\ln \frac{t}{r}\right) \underset{t<s<\infty}{\operatorname{essinf}} \varphi_{1}(x, s) \Phi^{-1}\left(t^{-n}\right) \frac{d t}{t} \leq C_{0} \varphi_{2}(x, r) \Phi^{-1}\left(r^{-n}\right),
$$

where $C_{0}$ does not depend on $x \in \mathbf{R}^{n}$ and $r>0$, then

$$
\left\|T_{b} f\right\|_{\mathcal{M}^{\Phi, \varphi_{2}}} \leq C\|b\|_{*}\|f\|_{\mathcal{M}^{\Phi, \varphi_{1}}},
$$

where $C$ does not depend on $f$ and $b$. 
Note that from Theorem 5.1, we recover the result obtained in [12] for the case $\Phi(u)=u^{p}$.

Theorem 5.2. Let $\Phi$ be a Young function with $1<a_{\Phi} \leq b_{\Phi}<\infty$ and $b \in$ $B M O\left(\mathbf{R}^{n}\right)$. Suppose that

$$
\int_{r}^{\infty}\left(1+\ln \frac{t}{r}\right) \varphi_{1}(x, t) \Phi^{-1}\left(t^{-n}\right) \frac{d t}{t} \leq C_{0} \varphi_{2}(x, r) \Phi^{-1}\left(r^{-n}\right),
$$

where $C_{0}$ does not depend on $x \in \mathbf{R}^{n}$ and $r>0$,

$$
\lim _{r \rightarrow 0} \frac{\ln \frac{1}{r}}{\Phi^{-1}\left(r^{-n}\right) \inf _{x \in \mathbf{R}^{n}} \varphi_{2}(x, r)}=0
$$

and

$$
c_{\delta}:=\int_{\delta}^{\infty}(1+|\ln t|) \sup _{x \in \mathbf{R}^{n}} \varphi_{1}(x, t) \frac{\Phi^{-1}\left(t^{-n}\right)}{t} d t<\infty
$$

for every $\delta>0$. Then the operator $T_{b}$ is bounded from $V \mathcal{M}^{\Phi, \varphi_{1}}\left(\mathbf{R}^{n}\right)$ to $V \mathcal{M}^{\Phi, \varphi_{2}}\left(\mathbf{R}^{n}\right)$.

5.2. Commutator $\mathfrak{M}^{b}$ of the maximal operator. We find it important to underline once again that the results of this subsection for the commutator $\mathfrak{M}^{b}$ of the maximal operator are obtained in supremal terms, i.e., under weaker assumptions than derived from more general theorems of Subsection 5.1. More precisely, the supremal condition (5.8) is weaker than the corresponding integral condition (5.1), see Remark 4.4 .

The following lemma contains a known extension of the property (2.3) from $L^{p_{-}}$ norms to Orlicz norms, see for instance [17], [16], where more general statements of rearrangement invariant spaces and also for variable exponent Lebesgue spaces may be found.

Lemma 5.3. Let $b \in B M O\left(\mathbf{R}^{n}\right)$ and $\Phi$ be a Young function of lower type $p_{0}$ and upper type $p_{1}, 1 \leq p_{0} \leq p_{1}<\infty$. Then

$$
\|b\|_{*} \approx \sup _{x \in \mathbf{R}^{n}, r>0} \Phi^{-1}\left(r^{-n}\right)\left\|b(\cdot)-b_{B(x, r)}\right\|_{L^{\Phi}(B(x, r))} .
$$

We also use the following lemma to prove our main estimates.

Lemma 5.4. For a Young function $\Phi$ and all balls $B$, the following inequality is valid

$$
\|f\|_{L^{1}(B)} \leq 2|B| \Phi^{-1}\left(|B|^{-1}\right)\|f\|_{L^{\Phi}(B)} .
$$

Proof. The proof is obtianed by applying Hölder inequality with Young functions and the known facts:

$$
\left\|\chi_{B}\right\|_{L^{\Phi}\left(\mathbf{R}^{n}\right)}=\frac{1}{\Phi^{-1}\left(|B|^{-1}\right)}
$$

and $r \leq \Phi^{-1}(r) \widetilde{\Phi}^{-1}(r) \leq 2 r$ for $r \geq 0$.

The following lemma is crucial for the main result of this section.

Lemma 5.5. Let $\Phi$ be a Young function with $1<a_{\Phi} \leq b_{\Phi}<\infty$ and $b \in$ $B M O\left(\mathbf{R}^{n}\right)$, then the inequality

$$
\left\|\mathfrak{M}^{b} f\right\|_{L^{\Phi}\left(B\left(x_{0}, r\right)\right)} \lesssim \frac{\|b\|_{*}}{\Phi^{-1}\left(r^{-n}\right)} \sup _{t>r}\left(1+\ln \frac{t}{r}\right) \Phi^{-1}\left(t^{-n}\right)\|f\|_{L^{\Phi}\left(B\left(x_{0}, t\right)\right)}
$$


holds for any ball $B\left(x_{0}, r\right)$ and for all $f \in L_{\mathrm{loc}}^{\Phi}\left(\mathbf{R}^{n}\right)$.

Proof. For $B=B\left(x_{0}, r\right)$, write $f=f_{1}+f_{2}$ with $f_{1}=f \chi_{2 B}$ and $f_{2}=f \chi_{\mathrm{c}_{(2 B)}}$, so that

$$
\left\|\mathfrak{M}^{b} f\right\|_{L^{\Phi}(B)} \leq\left\|\mathfrak{M}^{b} f_{1}\right\|_{L^{\Phi}(B)}+\left\|\mathfrak{M}^{b} f_{2}\right\|_{L^{\Phi}(B)} .
$$

By the boundedness of the operator $\mathfrak{M}^{b}$ in the space $L^{\Phi}\left(\mathbf{R}^{n}\right)$ provided by Theorem 2.6, we obtain

$$
\left\|\mathfrak{M}^{b} f_{1}\right\|_{L^{\Phi}(B)} \leq\left\|\mathfrak{M}^{b} f_{1}\right\|_{L^{\Phi}\left(\mathbf{R}^{n}\right)} \lesssim\|b\|_{*}\left\|f_{1}\right\|_{L^{\Phi}\left(\mathbf{R}^{n}\right)}=\|b\|_{*}\|f\|_{L^{\Phi}(2 B)} .
$$

For $x \in B$ we have

$$
\mathfrak{M}^{b} f_{2}(x)=\sup _{t>0} \frac{1}{|B(x, t)|} \int_{B(x, t) \cap^{\complement}(2 B)}|b(y)-b(x)||f(y)| d y .
$$

Note that if $B(x, t) \cap\left\{{ }^{\mathrm{C}}(2 B)\right\}=B(x, t) \backslash 2 B \neq \emptyset$, then $t>r$. Indeed, if $y \in$ $B(x, t) \backslash 2 B$, then $t>|x-y| \geq\left|x_{0}-y\right|-\left|x_{0}-x\right|>2 r-r=r$.

On the other hand, $B(x, t) \backslash 2 B \subset B\left(x_{0}, 2 t\right)$. Indeed, if $y \in B(x, t) \backslash 2 B$, then we get $\left|x_{0}-y\right| \leq|x-y|+\left|x_{0}-x\right|<t+r<2 t$. Hence

$$
\begin{aligned}
\mathfrak{M}^{b}\left(f_{2}\right)(x) & \leq \sup _{t>r} \frac{1}{\left|B\left(x_{0}, t\right)\right|} \int_{B\left(x_{0}, 2 t\right)}|b(y)-b(x)||f(y)| d y \\
& =2^{n} \sup _{t>2 r} \frac{1}{\left|B\left(x_{0}, t\right)\right|} \int_{B\left(x_{0}, t\right)}|b(y)-b(x)||f(y)| d y .
\end{aligned}
$$

Then

$$
\begin{aligned}
\left\|\mathfrak{M}^{b} f_{2}\right\|_{L^{\Phi}(B)} \lesssim & \left\|\sup _{t>2 r} \frac{1}{\left|B\left(x_{0}, t\right)\right|} \int_{B\left(x_{0}, t\right)}|b(y)-b(\cdot)||f(y)| d y\right\|_{L^{\Phi}(B)} \\
\lesssim J_{1}+J_{2}= & \left\|\sup _{t>2 r} \frac{1}{\left|B\left(x_{0}, t\right)\right|} \int_{B\left(x_{0}, t\right)}\left|b(y)-b_{B}\right||f(y)| d y\right\|_{L^{\Phi}(B)} \\
& +\left\|\sup _{t>2 r} \frac{1}{\left|B\left(x_{0}, t\right)\right|} \int_{B\left(x_{0}, t\right)}\left|b(\cdot)-b_{B}\right||f(y)| d y\right\|_{L^{\Phi}(B)} .
\end{aligned}
$$

For the term $J_{1}$ by $(5.5)$ we obtain

$$
J_{1} \approx \frac{1}{\Phi^{-1}\left(r^{-n}\right)} \sup _{t>2 r} \frac{1}{t^{n}} \int_{B\left(x_{0}, t\right)}\left|b(y)-b_{B}\right||f(y)| d y
$$

and split it as follows:

$$
\begin{aligned}
J_{1} \lesssim & \frac{1}{\Phi^{-1}\left(r^{-n}\right)} \sup _{t>2 r} \frac{1}{t^{n}} \int_{B\left(x_{0}, t\right)}\left|b(y)-b_{B\left(x_{0}, t\right)}\right||f(y)| d y \\
& +\frac{1}{\Phi^{-1}\left(r^{-n}\right)} \sup _{t>2 r} \frac{1}{t^{n}}\left|b_{B\left(x_{0}, r\right)}-b_{B\left(x_{0}, t\right)}\right| \int_{B\left(x_{0}, t\right)}|f(y)| d y
\end{aligned}
$$

Applying Hölder's inequality, by Lemmas 5.3 and 5.4 and (2.4) we get

$$
\begin{aligned}
J_{1} \lesssim & \frac{1}{\Phi^{-1}\left(r^{-n}\right)} \sup _{t>2 r} \frac{1}{t^{n}}\left\|b(\cdot)-b_{B\left(x_{0}, t\right)}\right\|_{L^{\tilde{\Phi}}\left(B\left(x_{0}, t\right)\right)}\|f\|_{L^{\Phi}\left(B\left(x_{0}, t\right)\right)} \\
& +\frac{1}{\Phi^{-1}\left(r^{-n}\right)} \sup _{t>2 r} \frac{1}{t^{n}}\left|b_{B\left(x_{0}, r\right)}-b_{B\left(x_{0}, t\right)}\right| t^{n} \Phi^{-1}\left(t^{-n}\right)\|f\|_{L^{\Phi}\left(B\left(x_{0}, t\right)\right)}
\end{aligned}
$$




$$
\lesssim \frac{\|b\|_{*}}{\Phi^{-1}\left(r^{-n}\right)} \sup _{t>2 r} \Phi^{-1}\left(t^{-n}\right)\left(1+\ln \frac{t}{r}\right)\|f\|_{L^{\Phi}\left(B\left(x_{0}, t\right)\right)} .
$$

For $J_{2}$ we obtain

$$
\begin{aligned}
J_{2} & \approx\left\|b(\cdot)-b_{B}\right\|_{L^{\Phi}(B)} \sup _{t>2 r} t^{-n} \int_{B\left(x_{0}, t\right)}|f(y)| d y \\
& \lesssim \frac{\|b\|_{*}}{\Phi^{-1}\left(r^{-n}\right)} \sup _{t>2 r} \Phi^{-1}\left(t^{-n}\right)\|f\|_{L^{\Phi}\left(B\left(x_{0}, t\right)\right)}
\end{aligned}
$$

gathering the estimates for $J_{1}$ and $J_{2}$, we get

$$
\left\|\mathfrak{M}^{b} f_{2}\right\|_{L^{\Phi}(B)} \lesssim \frac{\|b\|_{*}}{\Phi^{-1}\left(r^{-n}\right)} \sup _{t>2 r} \Phi^{-1}\left(t^{-n}\right)\left(1+\ln \frac{t}{r}\right)\|f\|_{L^{\Phi}\left(B\left(x_{0}, t\right)\right)} .
$$

To unite (5.7) with (5.6), observe that

$$
\frac{1}{\Phi^{-1}\left(r^{-n}\right)} \sup _{t>2 r} \Phi^{-1}\left(t^{-n}\right)\|f\|_{L^{\Phi}(B(x, t))} \geq C\|f\|_{L^{\Phi}(B(x, 2 r))},
$$

which completes the proof.

Theorem 5.6. Let $\Phi$ be a Young function with $1<a_{\Phi} \leq b_{\Phi}<\infty$ and $b \in$ $B M O\left(\mathbf{R}^{n}\right)$. If

$$
\sup _{r<t<\infty}\left(1+\ln \frac{t}{r}\right) \operatorname{ess~inf}_{t<s<\infty} \varphi_{1}(x, s) \Phi^{-1}\left(t^{-n}\right) \leq C_{0} \varphi_{2}(x, r) \Phi^{-1}\left(r^{-n}\right),
$$

where $C_{0}$ does not depend on $x \in \mathbf{R}^{n}$ and $r>0$, then

$$
\left\|\mathfrak{M}^{b} f\right\|_{\mathcal{M}^{\Phi, \varphi_{2}}} \leq C\|b\|_{*}\|f\|_{\mathcal{M}^{\Phi, \varphi_{1}}},
$$

where $C$ does not depend on $f$ and $b$.

Proof. Apply Lemma 5.5 and Theorem 2.3.

From this theorem we recover the result in [11] for the case $\Phi(u)=u^{p}$.

Theorem 5.7. Let $\Phi$ be a Young function with $1<a_{\Phi} \leq b_{\Phi}<\infty$ and $b \in$ $B M O\left(\mathbf{R}^{n}\right)$. Let us assume that $\varphi_{1}, \varphi_{1}, \Phi$ satisfy the conditions (5.3) and

$$
\sup _{r<t<\infty}\left(1+\ln \frac{t}{r}\right) \varphi_{1}(x, t) \Phi^{-1}\left(t^{-n}\right) \leq C_{0} \varphi_{2}(x, r) \Phi^{-1}\left(r^{-n}\right),
$$

where $C_{0}$ does not depend on $x \in \mathbf{R}^{n}$ and $r>0$. Suppose also that

$$
\sup _{\delta<t<\infty}(1+|\ln t|) \Phi^{-1}\left(t^{-n}\right) \sup _{x \in \mathbf{R}^{n}} \varphi_{1}(x, t)<\infty
$$

for every $\delta>0$. Then the operator $\mathfrak{M}^{b}$ is bounded from $V \mathcal{M}^{\Phi, \varphi_{1}}\left(\mathbf{R}^{n}\right)$ to $V \mathcal{M}^{\Phi, \varphi_{2}}\left(\mathbf{R}^{n}\right)$.

Proof. The proof follows the same lines as in Theorem 5.2 with the difference that now splitting of $r<t<\infty$ to $r<t<\delta_{0}$ and $\delta_{0}<t<\infty$ must be made with respect to $\sup _{r<t<\infty}$, not integration.

Corollary 5.8. Let $1<p<\infty, b \in B M O\left(\mathbf{R}^{n}\right)$ and the condition

$$
\sup _{t>r}\left(1+\ln \frac{t}{r}\right) \frac{\varphi_{1}(x, t)}{t^{\frac{n}{p}}} \leq C_{0} \frac{\varphi_{2}(x, r)}{r^{\frac{n}{p}}}
$$


be fulfilled, where $C_{0}$ does not depend on $x \in \mathbf{R}^{n}$ and $r>0$. Let us also set

$$
\lim _{r \rightarrow 0} \frac{r^{\frac{n}{p}} \ln \frac{1}{r}}{\inf _{x \in \mathbf{R}^{n}} \varphi_{2}(x, r)}=0
$$

and

$$
\sup _{\delta<t<\infty} \frac{1+|\ln t|}{t^{\frac{n}{p}}} \sup _{x \in \mathbf{R}^{n}} \varphi_{1}(x, t)<\infty
$$

for every $\delta>0$. Then the commutator $\mathfrak{M}^{b}$ is bounded from $V \mathcal{M}^{p, \varphi_{1}}\left(\mathbf{R}^{n}\right)$ to $V \mathcal{M}^{p, \varphi_{2}}\left(\mathbf{R}^{n}\right)$. In particular, this holds for the vanishing Morrey spaces $V \mathcal{M}^{p, \lambda}\left(\mathbf{R}^{n}\right)$ with $\varphi_{1}(x, r)=\varphi_{2}(x, r)=r^{\frac{\lambda}{p}}, 0 \leq \lambda<n$.

Proof. Let $\Phi(t):=t^{p}$ for all $t \in(0, \infty)$ with $p \in(1, \infty)$. Then $\Phi$ is a Young function with $a_{\Phi}=b_{\Phi}=p \in(1, \infty)$, and $\mathcal{M}^{\Phi, \varphi}=\mathcal{M}^{p, \varphi}$. Thus if we take $\Phi(t)=t^{p}$ at Theorem 5.7, after easy calculations we get Corollary 5.8.

In particular, this holds for the vanishing Morrey spaces $V \mathcal{M}^{p, \lambda}\left(\mathbf{R}^{n}\right)$ with $\varphi_{1}(x, r)=$ $\varphi_{2}(x, r)=r^{\frac{\lambda}{p}}, 0 \leq \lambda<n$. Indeed, if in the conditions (5.9), (5.10) and (5.11) we take $\varphi_{1}(x, r)=\varphi_{2}(x, r)=r^{\frac{\lambda}{p}}, 0 \leq \lambda<n$, then we have

$$
\begin{aligned}
& \sup _{t>r}\left(1+\ln \frac{t}{r}\right) t^{\frac{\lambda-n}{p}} \leq C_{0} r^{\frac{\lambda-n}{p}} \Longleftrightarrow \sup _{t>1}(1+\ln t) t^{\frac{\lambda-n}{p}} \leq C_{0}, \\
& \lim _{r \rightarrow 0} r^{\frac{n-\lambda}{p}} \ln \frac{1}{r}=0 \quad \text { and } \sup _{\delta<t<\infty}(1+|\ln t|) t^{\frac{\lambda-n}{p}}<\infty .
\end{aligned}
$$

Acknowledgements. The authors thank the anonymous referees for careful reading of the paper and very useful comments.

\section{References}

[1] Akbulut, A., V.S. Guliyev, and R. Mustafayev: On the boundedness of the maximal operator and singular integral operators in generalized Morrey spaces. - Math. Bohem. 137:1, 2012, 27-43.

[2] Burenkov, V., A. Gogatishvili, V. S. Guliyev, and R. Mustafayev: Boundedness of the fractional maximal operator in local Morrey-type spaces. - Complex Var. Elliptic Equ. 55:8-10, 2010, 739-758.

[3] Calderon, A. P.: Commutators of singular integral operators. - Proc. Nat. Acad. Sci. U.S.A. 53, 1965, 1092-1099.

[4] Cianchi, A.: Strong and weak type inequalities for some classical operators in Orlicz spaces. - J. London Math. Soc. 60:1, 1999, 187-202.

[5] Coifman, R., P.-L. Lions, Y. Meyer, and S. Semmes: Compensated compactness and Hardy spaces. - J. Math. Pures Appl. 72, 1993, 247-286.

[6] Coifman, R. R., R. Rochberg, and G. Weiss: Factorization theorems for Hardy spaces in several variables. - Ann. of Math. (2) 103:3, 1976, 611-635.

[7] Deringoz, F., V.S. Guliyev, and S. Samko: Boundedness of maximal and singular operators on generalized Orlicz-Morrey spaces. - In: Operator Theory, Operator Algebras and Applications, Oper. Theory Adv. Appl. 242, 2014, 139-158.

[8] Fu, X., D. YANG, and W. YuAN: Boundedness of multilinear commutators of CalderónZygmund operators on Orlicz spaces over non-homogeneous spaces. - Taiwanese J. Math. 16, 2012, 2203-2238. 
[9] Garcia-Cuerva, J., E. Harboure, C. Segovia, and J. L. Torrea: Weighted norm inequalities for commutators of strongly singular integrals. - Indiana Univ. Math. J. 40:4, 1991, 1397-1420.

[10] Genebashvili, I., A. Gogatishvili, V. Kokilashvili, and M. Krbec: Weight theory for integral transforms on spaces of homogeneous type. - Longman, Harlow, 1998.

[11] Guliyev, V.S., A. Akbulut, and Y. Y. Mammadov: Boundedness of fractional maximal operator and their higher order commutators in generalized Morrey spaces on Carnot groups. - Acta Math. Sci. Ser. B Engl. Ed. 33:5, 2013, 1329-1346.

[12] Guliyev, V.S., S. S. Aliyev, T. Karaman, and P. S. Shukurov: Boundedness of sublinear operators and commutators on generalized Morrey space. - Integr. Equ. Oper. Theory 71:3, 2011, 327-355.

[13] Guliyev, V.S., F. Deringoz, and J. J. Hasanov: $\Phi$-admissible singular operators and their commutators on vanishing generalized Orlicz-Morrey spaces. - J. Inequal. Appl. 2014:143, 2014, $1-18$.

[14] Guliyev, V. S., and L. Softova: Global regularity in generalized Morrey spaces of solutions to nondivergence elliptic equations with VMO coefficients. - Potential Anal. 38:4, 2013, 843862.

[15] Guliyev, V.S., and L. Softova: Generalized Morrey regularity for parabolic equations with discontinuity data. - Proc. Edinburgh Math. Soc. (to appear).

[16] Ho, K.-P.: Characterization of BMO in terms of rearrangement-invariant Banach function spaces. - Expo. Math. 27, 2009, 363-372.

[17] IzUKI, M., and Y. SAwano: Variable Lebesgue norm estimates for BMO functions. - Czechoslovak Math. J. 62:3, 2012, 717-727.

[18] Kita, H.: On maximal functions in Orlicz spaces. - Proc. Amer. Math. Soc. 124, 1996, 30193025.

[19] KitA, H.: On Hardy-Littlewood maximal functions in Orlicz spaces. - Math. Nachr. 183, 1997, 135-155.

[20] Kokilashvili, V., and M. M. KrbeC: Weighted inequalities in Lorentz and Orlicz spaces. World Scientific, Singapore, 1991.

[21] Krasnoselskit, M. A., and Ya. B. Rutickir: Convex functions and Orlicz spaces. - P. Noordhoff Ltd., Groningen, 1961 (English translation).

[22] Mizuta, Y., E. Nakai, T. Ohno, and T. Shimomura: Maximal functions, Riesz potentials and Sobolev embeddings on Musielak-Orlicz-Morrey spaces of variable exponent in $R^{n}$. - Rev. Mat. Complut. 25:2, 2012, 413-434.

[23] Morrey, C. B.: On the solutions of quasi-linear elliptic partial differential equations. - Trans. Amer. Math. Soc. 43, 1938, 126-166.

[24] NAKAI, E.: Generalized fractional integrals on Orlicz-Morrey spaces. - In: Banach and Function Spaces. (Kitakyushu, 2003), Yokohama Publishers, Yokohama, 2004, 323-333.

[25] NAKAI, E.: Orlicz-Morrey spaces and the Hardy-Littlewood maximal function. - Studia Math. $188: 3,2008,193-221$.

[26] NAKAI, E.: Calderón-Zygmund operators on Orlicz-Morrey spaces and modular inequalities. - In: Banach and function spaces II, Yokohama Publ, Yokohama, 2008, 393-410.

[27] Persson, L. E., M. A. Ragusa, N. Samko, and P. Wall: Commutators of Hardy operators in vanishing Morrey spaces. - AIP Conf. Proc. 1493, 2012, 859.

[28] Ragusa, M. A.: Commutators of fractional integral operators on vanishing-Morrey spaces. J. Global Optim. 40:1-3, 2008, 361-368.

[29] Rao, M. M., and Z. D. Ren: Theory of Orlicz spaces. - M. Dekker, Inc., New York, 1991. 
[30] SAmko, N.: Maximal, potential and singular operators in vanishing generalized Morrey spaces. - J. Global Optim. 57:4, 2013, 1385-1399.

[31] Samko, S.: Morrey spaces are closely embedded between vanishing Stummel spaces. - Math. Inequal. Appl. 17:2, 2014, 627-639.

[32] SaWAno, Y.: A vector-valued sharp maximal inequality on Morrey spaces with nondoubling measures. - Georgian Math. J. 13:1, 2006, 153-172.

[33] Sawano, Y.: Generalized Morrey spaces for non-doubling measures. - NoDEA Nonlinear Differential Equations Appl. 15, 2008, 413-425.

[34] Sawano, Y., S. Sugano, and H. Tanaka: Orlicz-Morrey spaces and fractional operators. Potential Anal. 36:4, 2012, 517-556.

[35] Softova, L. G.: Singular integrals and commutators in generalized Morrey spaces. - Acta Math. Sin. (Engl. Ser.) 22:3, 2006, 757-766.

[36] Vitanza, C.: Functions with vanishing Morrey norm and elliptic partial differential equations. - In: Proceedings of Methods of Real Analysis and Partial Differential Equations, Capri, Springer, 1990, 147-150.

[37] Vitanza, C.: Regularity results for a class of elliptic equations with coefficients in Morrey spaces. - Ric. Mat. 42:2, 1993, 265-281.

Received 5 August $2014 \bullet$ Accepted 27 October 2014 